\title{
LA GESTIÓN DE LA EDUCACIÓN AMBIENTAL EN LAS ORGANIZACIONES DESDE LA PERSPECTIVA DE LOS ESTUDIANTES DE LA UNIVERSIDAD POLITÉCNICA DEL VALLE DEL ÉVORA, MÉXICO
}

\author{
J. E. Olaguez ${ }^{1}$, E. Peña ${ }^{2}$, P. Espino ${ }^{3}$ \\ ${ }^{1}$ Programa Académico Administración y Gestión de Pequeñas y Medianas Empresas, Universidad Politécnica del \\ Valle del Évora, México; ${ }^{2}$ Universidad de las Tunas, Cuba, ${ }^{3}$ Unidad académica de Ingeniería Mecatrónica, \\ Universidad Politécnica de Sinaloa, México \\ eolaguezt@gmail.com ${ }^{1}$
}

Recepción 30/10/2016 - Aprobación 12/12/2017

DOI: 10.15628/holos.2017.5299

\section{RESUMEN}

La crisis ambiental, abarca no sólo cuestiones relacionadas con el agotamiento de los recursos, el efecto invernadero, la contaminación y el desequilibrio general de los ecosistemas, sino también las desigualdades económicas, la pobreza, la equidad y la justicia social. Por ello la búsqueda de un ecosistema equilibrado está cada vez más presente en diversos sectores de la sociedad; en este estudio se abordará lo que corresponde a la percepción de la educación ambiental para ayudar en la toma de conciencia emergente. Las instituciones de educación superior no deberían, permanecer ajenas a la solución de los problemas ambientales, pudiendo ser partícipes a través de sus funciones principales de docencia, investigación y vinculación. En este trabajo se analiza la percepción de estudiantes de nivel licenciatura de la Universidad Politécnica del Valle del Évora, México; con respecto a la educación ambiental y el impacto de la misma en su entorno. Se analizó el aspecto teórico y generalidades sobre la percepción de la educación ambiental, a través de un instrumento de recopilación de datos de tipo cuestionario, integrado por 26 preguntas en escala de Likert, a 115 estudiantes. El análisis de contenido de las respuestas permite analizar las actitudes y valoración de los estudiantes con respecto a los conceptos ecología, medio ambiente y la relación del ambiente con la ciencia y tecnología. Se concluye que es necesario incluir en los planes curriculares de la Universidad Politécnica del Valle del Évora, México; el tema de educación ambiental desde un enfoque transversal.

PALABRAS-CLAVE: Educación ambiental, cultura, estudiantes, organizaciones, sustentabilidad

\section{MANAGEMENT OF ENVIRONMENTAL EDUCATION ORGANIZATIONS FROM THE PERSPECTIVE OF STUDENTS OF THE POLYTECHNIC UNIVERSITY OF THE VALLEY OF ÉVORA, MÉXICO}

\section{ABSTRACT}

Regarding the environmental crisis, which covers not only issues related to resource depletion, greenhouse effect, pollution and the general imbalance of ecosystems, but also economic inequalities, poverty, equity and social justice. Therefore, the search for a balanced ecosystem is increasingly present in various sectors of society, in this study will address what corresponds to the perception of environmental education to assist in emerging awareness. It is considered that higher education institutions cannot, and should, remain outside the solution of environmental problems through their core functions of teaching, research and links. In this paper the perception of under graduate students from the Polytechnic University of the Évora Valley, Mexico; is addressed with regard to environmental education and the impact of it on their environment. The theoretical aspect and generalities about the perception of environmental education, was analyzed through a data collection instrument type questionnaire composed of 26 questions in Likert scale, to 115 students. The content analysis of responses to analyze the attitudes and assessment of students regarding the concepts ecology, environment and environmental relationship with science and technology. It is concluded that it is necessary to include in the curricula plan of the Polytechnic University of the Valley of Évora, from a cross-cutting approach the subject of environmental education. 


\section{INTRODUCCIÓN}

La búsqueda de un equilibrio del entorno se hace presente en varios sectores de la sociedad, pues a más de cuarenta años de la Conferencia de las Naciones Unidas sobre el médio ambiente humano celebrada en Estocolmo (1972), se siguen haciendo pronunciamientos sobre la emergente necesidad de detener el deterioro ecológico, sin embargo las acciones reales para un cambio radical se han manifestado aletargadamente, por ello en el presente estudio se aborda la percepción de la educación ambiental desde la óptica de estudiantes de nivel superior, estudiantes de la Universidad Politécnica del Valle del Évora, México, con el propósito de contribuir crítica y analíticamente en la gestión y tratamiento de la Educación Ambiental, identificar las implicaciones principales al fomentar y promover la educación ambiental en la universidad, proponer alternativas para fomentar de manera permanente y relevante la educación ambiental dentro de la universidad e incidir de manera consistente en la solución de la problemática ambiental que se vive en la actualidad.

Para la consolidación de una perspectiva ambiental de desarrollo, es de vital importancia la participación de todos los sectores e instituciones sociales, y particularmente las instituciones de educación superior. El potencial que tienen las instituciones de educación superior demanda que asuman una mayor responsabilidad, fomenten y promuevan en sus programas académicos y de manera transversal la educación ambiental, y poder coadyuvar a mejorar la calidad de vida en el planeta. Se considera que las instituciones educativas pueden contribuir de manera estratégica a mejorar la problemática ambiental, a través de sus funciones principales de docencia, investigación y vinculación.

Como lo señala Bravo (2009), quien menciona que las Instituciones de Educación Superior en México (IES), cuentan con un gran potencial ya que se centran alrededor del conocimiento, sobre el cual giran sus actividades fundamentales: enseñanza, investigación y difusión; en todas las áreas del conocimiento. Por su parte Medina (2009), describe que esencialmente los niveles educativos superiores se les atribuye la responsabilidad de desarrollar las capacidades de los individuos para coadyuvar a mejorar la calidad de vida en el planeta. Ya que esta tiene la posibilidad de incidir en la formación de los individuos integrando la dimensión ambiental como una prioridad que guíe su desempeño personal y profesional en cualquiera de sus campos de desarrollo. Por tal motivo las IES, deben de incorporar la dimensión ambiental en los planes de desarrollo institucional, incluyendo acciones de transmisión, generación, aplicación y difusión del conocimiento, así como sistemas de manejo ambiental para el uso eficiente y ahorro de los recursos institucionales (ANUIES, 2009).Las IES han tenido crecientes aportaciones a la solución de la problemática ambiental, ya sea a través de la docencia, la investigación o difusión, sin embargo, se requiere esfuerzos integrales para lograr un mayor impacto.

Para dar sustento a este trabajo se cita el concepto de medio ambiente y de educación ambiental (EA). La Ley General del Equilibrio Ecológico y Protección al Ambiente (LGEEPA, 2008), en su artículo 3ro. Párrafo I, define como Ambiente: "El conjunto de elementos naturales y artificiales o inducidos por el hombre que hacen posible la existencia y desarrollo de los seres humanos y demás organismos vivos que interactúan en un espacio y tiempo determinado". En esa perspectiva se sitúa la educación ambiental, como un movimiento transformador que se desarrolló en los años setenta y promovido por la Unesco (2010) y el Pnuma (2009), así como 
también por instituciones públicas y privadas. Su objetivo esencial es propiciar un cambio en los valores y formas de vida que responda al doble reto (ecológico y social) que tienen planteadas nuestras sociedades (Novo y Bautista, 2012). Para Ávila (2009), educación ambiental es el proceso de adquisición de valores y clarificación de conceptos cuyo objetivo es desarrollar actitudes y capacidades necesarias para entender y apreciar las interrelaciones entre el hombre, su cultura y su entorno biofísico.

Bedoy (2009), plantea que la educación ambiental es entendida como un proceso integral, político, pedagógico, social, orientado a conocer y comprender la esencia de la situación ambiental, para propiciar la participación activa y organizada de la población en la transformación de su realidad, en función de un proyecto de sociedad ambientalmente sustentable y socialmente justas. González (2001), señala que los objetivos de la educación ambiental están implícitos en las propuestas ambientales los cuales son: a) Lograr que los actores sociales, en forma individual o colectiva, comprendan la naturaleza compleja del medio ambiente natural, así como del creado por el hombre, que es el resultado de la interacción de sus aspectos biológicos, físicos, sociales, económicos y culturales. b) Adquirir los valores, la conducta, cambio de cultura, comportamiento y habilidades prácticas para prevenir y solucionar los problemas ambientales.

De acuerdo a Olaguez y Espino (2013), se pretende lograr un cambio conceptual de la relación humano-naturaleza, a través de la educación ambiental, teniendo el propósito de que las personas adquieran conciencia de su entorno y puedan realizar cambios en sus valores, conductas y estilos de vida, así como ampliar sus conocimientos para impulsar los procesos de prevención y resolución de los problemas ambientales presentes y futuros, y a su vez la educación ambiental sea la herramienta fundamental para que todas las personas adquieran conciencia de su entorno.

Existen estudios previos respecto a la percepción de los estudiantes hacia el medio ambiente. Por ejemplo, Sosa et al. (2008), presentó un estudio donde se evalúa la percepción ambiental en estudiantes universitarios, considerando como indicadores ambientales, lo social, económico y ambiental. El estudio mostró que la conducta pro ambientalista del estudiante universitario está definida a partir de valores, creencias, actitudes ambientales y que probablemente estas sean influenciadas por el área académica en la que el estudiante se vea involucrado.

Zamorano et al. (2009), realizó una investigación para indagar en un grupo de estudiantes la opinión que tienen sobre el medio ambiente así como también sobre los problemas ambientales e identificar el conocimiento de la problemática que afecta a su región, su estudió determino que los estudiantes entienden la necesidad del aprovechamiento racional de la naturaleza, su percepción ambiental es real, en relación con la intervención del ser humano en la naturaleza, comprende la problemática ambiental. Otro análisis es presentado por Sosa et al. (2010), muestra la situación que guarda la educación ambiental en el nivel superior, el estudio consistió en la utilización de métodos cuantitativos y cualitativos para diagnosticar el grado de cultura ambiental en los estudiantes, los resultados del estudio muestran un bajo nivel de cultura ambiental, señala que el contexto institucional opera como un factor que inhibe la educación ambiental debido a que la formación ambiental no es considerada prioritaria. Por su parte la investigación presentada por Márquez et al. (2011) indica que los estudiantes poseen un nivel de 
cultura ambiental bajo y carecen de conocimientos y habilidades necesarias para realizar cambios ambientales favorables en sus estilos de vida, el estudio permitió identificar actitudes positivas en los estudiantes para aprender prácticas sustentables, así como la importancia que tiene la escuela como fuente de información ambiental.

En el estudio realizado por García et al. (2011), muestra los resultados del estudio sobre los conocimientos, actitudes, creencias y comportamientos de los estudiantes y profesores acerca de la gestión ambiental del agua, energía y la producción de residuos. Los resultados obtenidos señalan que no se deduce una mejora progresiva de sus conocimientos y actitudes en función del nivel educativo cursado. Los estudiantes se declaran ser muy conscientes, sin embargo, sus actuaciones no son coherentes con una participación activa en la solución de los problemas.

Barazarte y Neaman (2013), evaluaron el efecto del conocimiento ambiental en el comportamiento pro-ambiental de los estudiantes de la enseñanza media así como también el de analizar el efecto del grupo socioeconómico y el tipo de dependencia del colegio en el conocimiento ambiental y el comportamiento pro-ambiental de los estudiantes, sus resultados señalan que el conocimiento ambiental no incide en el comportamiento pro-ambiental de los estudiantes de los colegios que conformaron la muestra, además que el grupo socioeconómico y el tipo de dependencia del colegio inciden en el conocimiento ambiental y en el comportamiento pro-ambiental delos estudiantes.

El estudio realizado por Calafell y Bonil (2014), permitió identificar patrones de los profesionales según sus concepciones de medio ambiente que participan en actividades de formación para poder orientar el cambio en su acción docente. Por su parte el estudio realizado por Espino et al. (2015) pone en evidencia la importancia que los estudiantes le otorgan al medio ambiente, la contaminación y destrucción de los recursos naturales son percibidos como los principales problemas del entorno en el que viven. Además, consideran que tanto la ciencia y la tecnología son consideraros como la causa y la solución de los problemas del medio ambiente.

Por lo anterior, es de suma importancia conocer la percepción que tienen los estudiantes de la universidad con respecto a temas ambientales y problemáticas que ocurren desde las perspectivas locales y globales. En este sentido es importante conocer si los estudiantes tienen, las actitudes, valores, conductas responsables y respetuosas con el ambiente. Así como también definir si éstos cuentan con la suficiente educación ambiental para enfrentar los nuevos retos de un mundo globalizado.

\section{METODOLOGÍA}

Se aplicó un instrumento consistente en un cuestionario compuesto de 26 preguntas y afirmaciones de las variables a medir. Se valoró la percepción de los estudiantes sobre aspectos relacionados con la educación ambiental; su concepto, métodos adecuados para lograr su efectividad, actores sociales encargados de desarrollarla, entre otros aspectos. La investigación se desarrolló en la Universidad Politécnica del Valle del Évora, México. La población estuvo integrada por estudiantes de los tres programas académicos que allí se imparten, de los cuatrimestres 5 to y 8 vo respectivamente. Se calculó el tamaño de muestra necesario para lograr 
confiabilidad en las respuestas respecto a la población total de la ecuación (1).

$$
n=\frac{Z^{2}[N(p)(d)]}{e^{2}(N-1)+Z^{2}(p)(q)}
$$

\section{Donde:}

$\mathrm{n}$ : tamaño de la muestra requerida.

Z: Valor estándar de Z=1,96 para un nivel de confiabilidad del $95 \%$.

$\mathrm{N}$ : Tamaño de la población (121 estudiantes de los cuatrimestres 5to y 8vo).

p: Variabilidad positiva (probabilidad de valor esperado $=0,5$ )

q: Variabilidad negativa (probabilidad de valor no esperado $q=1-p$ )

e: Error máximo permitido asumido (5\%)

En base a la ecuación anterior se calculó un tamaño de muestra de 115encuestas aplicadas a estudiantes de las tres especialidades. Para la aplicación del instrumento se empleó la Herramienta Google Forms y para el análisis de los resultados se emplearon la propia herramienta mencionada anteriormente, así como la aplicación Microsoft Excel. La muestra estuvo conformada por un $43 \%$ de estudiantes de Licenciatura en Administración de Pequeñas y Medianas Empresas; 30 \% de estudiantes de la especialidad de Ingeniero en Agrotecnología y el $27 \%$ estudia Ingeniería en Sistemas Computacionales. Estos valores representan respecto a la matrícula total de la Universidad el $15.9 ; 11.1$ y $10 \%$ en el mismo orden en que fueron mencionados anteriormente. Respecto a género, estuvieron representados en un 45 y $55 \%$ para el femenino y masculino respectivamente (ver figura 1 ).

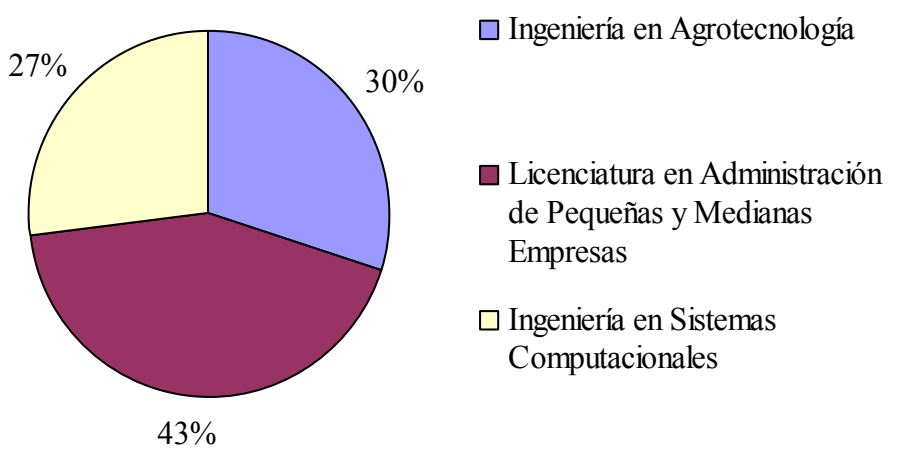

Figura 1: Distribución porcentual de la muestra por especialidad.

La muestra se conformó por estudiantes residentesde20 localidades de la Región del Valle del Évora (tabla 1) que incluye los municipios Angostura, Mocorito, Navolato y Guasave. Se destaca por su alta representatividad el poblado La Reforma con un $29 \%$; en segundo orden con valores entre el 10 y el $15 \%$ el poblado Leopoldo Sánchez Celis y las Colonias Agrícolas Melchor Ocampo, México e Independencia. Los demás estuvieron representados en porcentajes entre el 1 y $5 \%$.Entre los municipios los de mayor representatividad fueron Angostura con el $79 \%$, mientras el $18 \%$ reside en Mocorito. Se computaron además respuestas de estudiantes 
residentes en localidades de Guasave y Navolato, por lo que toda la región del Valle del Évora estuvo representada.

Tabla 1: Localidades de residencia de los encuestados.

\begin{tabular}{|c|c|c|c|c|c|}
\hline No. & Localidad y Municipio & $\%$ & No. & Localidad y Municipio & $\%$ \\
\hline 1 & La Reforma, Angostura & 29 & 11 & Alhuey, Angostura & 1 \\
\hline 2 & $\begin{array}{l}\text { Poblado Leopoldo Sánchez, } \\
\text { Angostura }\end{array}$ & 14 & 12 & Angostura, Angostura & 1 \\
\hline 3 & Colonia Melchor Ocampo, Mocorito & 12 & 13 & Batury, Angostura & 1 \\
\hline 4 & Colonia Agrícola México, Angostura & 11 & 14 & Ejido Bruno B. García. Angostura & 1 \\
\hline 5 & $\begin{array}{l}\text { Colonia Agrícola Independencia, } \\
\text { Angostura }\end{array}$ & 10 & 15 & Carretera Costera, Angostura & 1 \\
\hline 6 & Potrero de los Sánchez, Mocorito & 5 & 16 & Ejido Rafael Buelna, Angostura & 1 \\
\hline 7 & Colonia Ensenada, Navolato & 3 & 17 & $\begin{array}{l}\text { Ejido Valentín Gómez Farisd, } \\
\text { Angostura }\end{array}$ & 1 \\
\hline 8 & Costa Azul, Angostura & 3 & 18 & Guasave, Guasave & 1 \\
\hline 9 & Colonia Agrícola Sinaloa , Angostura & 2 & 19 & La Esperanza, Angostura & 1 \\
\hline 10 & Ejido Ignacio Allende, Angostura & 2 & 20 & Zapotillo, Mocorito & 1 \\
\hline
\end{tabular}

Los resultados de la encuesta fueron procesados mediante el empleo de herramientas de estadística matemática. Se calcularon las frecuencias absolutas y relativas de cada respuesta. En la primera sección de la encuesta cuando se estudió la variable nivel de conocimientos sobre aspectos relacionados con el medio ambiente se aplicó el test de estadística no paramétrica chi cuadrado de independencia para comprobar si existía alguna relación entre la especialidad y/o el género con el nivel de conocimientos declarado sobre los diferentes temas a los que se refirió la encuesta.

Las Hipótesis probadas fueron:

$\mathrm{H}_{0}$ : Las variables son independientes

$\mathrm{H}_{1}$ : Las variables no son independientes

- Si p menor o igual que 0,05 el resultado no es significativo, es decir, se acepta la hipótesis nula de independencia y por lo tanto se concluye que ambas variables estudiadas son independientes, no existe una relación entre ellas.

- Si p mayor que 0,05 el resultado es significativo, es decir, se rechaza la hipótesis nula de independencia y por lo tanto se concluye que ambas variables estudiadas son dependientes, existe una relación entre ellas.

En la segunda sección de la encuesta, se valoró la percepción de los estudiantes sobre aspectos relacionados con la educación ambiental. Se calculó el coeficiente de Kendall para evaluar el grado de concordancia en las respuestas. Se plantearon las hipótesis.

$\mathrm{H}_{0}$ : No existe asociación entre las calificaciones de todos los encuestados y el estándar conocido. $\mathrm{H}_{1}$ : Las calificaciones de todos los encuestados se asocian con el estándar conocido. El valor $\mathrm{p}$ provee la probabilidad de obtener su muestra, con su coeficiente de correlación de Kendall 
particular, si la hipótesis nula $\left(\mathrm{H}_{0}\right)$ es verdadera. Si el valor $\mathrm{p}$ es menor o igual a un nivel predeterminado de significancia (nivel $\alpha$ ), se rechaza la hipótesis nula y da crédito a la alternativa. Este coeficiente se calcula mediante la ecuación (2):

$W=\frac{S}{1 / 12 K^{2}\left(n^{3}-n\right)}$

W: Coeficiente de concordancia.

$\mathrm{K}$ : Cantidad de encuestados.

$\mathrm{n}$ : Cantidad de variables.

T: Factor de corrección.

S: Suma de los cuadrados de las desviaciones.

La significación del coeficiente de Kendal se evaluó mediante el estadígrafo $\chi^{2}$ y el $p$ valor.

\section{RESULTADOS Y DISCUSIÓN}

A continuación, se muestran resultados de la percepción sobre aspectos relacionados con la educación ambiental. El primer aspecto sobre el que se preguntó tuvo que ver con la edad en que deben comenzar a desarrollarse los preceptos de educación ambiental. Se refieren a continuación algunos de los principales planteamientos referidos. En la mayoría de ellos se coincide que deben trabajarse desde la niñez. Se presentan las respuestas dadas por los encuestados acerca del momento en que debe iniciarse la educación (se respetó la redacción original de los encuestados).

- Tres años.

- Cualquier momento.

- Cuando llevas a cabo una responsabilidad por ti mismo para cuidar el medio ambiente.

- Cuando nos presentamos ante una situación que nos afecta a causa del medio ambiente.

- Desde bebés.

- Desde educación pre-escolar.

- Desde el hogar.

- Desde el nivel prescolar.

- Desde este mismo momento para poner en práctica todas y cada una de las actividades que son necesarias desempeñar para conservar un ambiente sano.

- Desde la infancia, ya sea en la familia o instituto educativo.

- Desde los primeros años de vida, los padres deben inculcar en sus hijos el sentido de la protección ambiental.

- Desde niño (siete años).

- Desde niño, en cuanto tenga la capacidad de razonar.

- Desde niños en nuestra casa.

- Desde pequeños para inculcar valores ambientales y el amor por cuidar el planeta.

- Desde pequeños, en el momento que adquirimos cualquier otro conocimiento.

- Desde que comienza la educación de la persona.

- Desde que el individuo tiene la capacidad de razonar, desde corta edad. 
- Desde que el niño empieza a relacionarse con su medio ambiente y tenga la debida conciencia de conocer las ventajas de cuidarlo.

- Desde que empiezas a contaminar.

- Desde siempre, uno debe tener conciencia sobre esto todo el tiempo.

- Desde temprana edad (5 años).

- Desde ya, o desde antes.

- El conocimiento o la educación al medio ambiente debe comenzar desde que la persona nace o bien cuando ya empieza a razonar se le debe dar la educación.

- El cuidado del medio ambiente debe empezar desde que la persona nace, para que crezca con esa educación que hoy en día los padres no les enseñan a sus hijos.

- En el momento antes que este problema no tenga solución.

- En estos momentos (ahora).

- Entre más pronto mejor.

- Futuro.

- Hoy.

- Lo más pronto posible; ya o desde que vamos adquiriendo conocimientos sobre el entorno.

- Principalmente despertar interés desde escuelas primarias, llevar a un segundo nivel cuando se curse secundaria, y posteriormente en las universidades, de esta manera los alumnos que lleguen a nivel universidad poco recordarán sobre la importancia de la educación ambiental y así despertará de nuevo el conocimiento y la preocupación, debido a la etapa de desarrollo joven-adulto que está sufriendo el individuo.

- Todos los días se necesita adquirir conocimientos nuevos para la educación ambiental.

- Todo queda en la práctica.

- Todos debemos ya saber.

- Yo creo que no hay mal momento para adquirir los conocimientos sobre la educación ambiental.

Los rasgos generales encontrados en los planteamientos anteriores son:

- en algunos casos se refieren a edades (bebés, 3, 5 ó 7 años), mientras en otros a los niveles de enseñanza (preescolar y primaria).

- algunas respuestas supeditan la edad o el nivel de enseñanza al desarrollo biológico o la acción social (desde que se adquieren los primeros conocimientos, se tiene capacidad de razonar o se comienza a interactuar con el ambiente.

- otros se refieren a la edad de conjunto con el escenario (escuela, hogar o ambas).

- Se observan respuestas que se relacionan con el impacto que se genere, por ejemplo "desde que se empiece a contaminar".

- en algunos casos ubican la educación ambiental como tema del presente (desde ya), pasado, futuro o como algo permanente (desde que se nace y no debe cesar).

- de forma general la mayoría de las respuestas enmarcan como momento para comenzar la educación ambiental desde la niñez y la ven como un proceso continuo.

Acerca de la tipología de materias en las cuales debe tratarse la educación ambiental en los centros de enseñanza, en el $76 \%$ de las respuestas se cita la ecología, seguido de un $11 \%$ que las ubica en conocimientos de biología. El resto de las respuestas (en el rango del 1 al $10 \%$ ) los 
relacionan con la geografía, química, sociología entre otras materias (ver tabla 2).

Tabla 2. Percepción sobre tipología de materias para el tratamiento de la educación ambiental.

\begin{tabular}{ll}
\hline Materia & $\%$ \\
\hline Ecología & 76,0 \\
Biología & 11,0 \\
Sociología & 5,0 \\
Geografía & 4,0 \\
Química & 2,0 \\
Biología y Geografía & 1,0 \\
Todas & 1,0 \\
\hline
\end{tabular}

En consecuencia, con el análisis sobre el grado de confianza en diferentes actores sociales para desarrollar acciones en pro del mejoramiento ambiental y en el momento en que debe comenzar a desarrollarse la educación ambiental, se observó correspondencia cuando se les preguntó acerca de qué institución o instituciones deben fomentar la educación ambiental (tabla 3).Las respuestas mayoritariamente fueron: Todos (40\%) y la familia (23\%). El $19 \%$ dio como opción "la familia, instituciones educativas y medios de comunicación"; Un 4 \% respondió las instituciones educativas como opción única, mientras un $3 \%$ destaca que debe ser tarea de estas de conjunto con la familia. Para otro $5 \%$ la labor debe desarrollarse por la familia en conjunción con los medios de comunicación. Un 3 \% señala a las empresas, comercios y negocios, el $2 \%$ responsabiliza a los medios de comunicación de todo tipo e igual porcentaje a estos en unión a las instituciones educativas y el $1 \%$ indica que es responsabilidad de los gobiernos a todos los niveles.

Tabla 3. Percepción sobre el papel de las instituciones sociales en la educación ambiental.

\begin{tabular}{l|l}
\hline Respuesta & $\%$ \\
\hline Todos & 40,0 \\
La familia & 23,0 \\
La familia, instituciones educativas y medios de comunicación & 19,0 \\
La familia y los medios de comunicación & 5,0 \\
Instituciones educativas & 4,0 \\
Empresas comercios y negocios & 3,0 \\
La familia e instituciones educativas & 3,0 \\
Instituciones educativas y medios de comunicación & 2,0 \\
Gobiernos a todos los niveles & 1,0 \\
\hline
\end{tabular}

A continuación, la encuesta incluyó varios ítems con los que se pudo conocer la percepción de los estudiantes sobre diversos aspectos relacionados con la educación ambiental. Se les pidió valoraran en una escala del 1 al 5 en la que uno (1) significó "totalmente en desacuerdo" y cinco (5) "totalmente de acuerdo" en las interpelaciones siguientes:

- P16-Ante los problemas ambientales ¿Considera usted que la educación ambiental debería ser una asignatura en todos los niveles de enseñanza?

- P17-Todas las personas deberían adquirir una sólida educación ambiental. 
- P18-Para resolver los problemas de un país relacionados con la protección del medio ambiente, ¿¿ss preferible invertir en innovación tecnológica y ciencia antes que en educación ambiental?

- P19-Creo que la educación ambiental puede resolver los problemas relacionados con la protección del medio ambiente.

- P20-Debe incluirse la educación ambiental en la enseñanza obligatoria, aunque presuponga dejar de recibir contenidos de cálculo, física, lenguas u otros más a tono con la preparación profesional.

- P22-La cultura debe considerarse importante para el desarrollo de la educación ambiental.

- P23-La educación ambiental debe considerarse como un eje transversal en los contenidos, con sus actividades, de los programas o currículos escolares.

Los resultados indican mayoritariamente una alta sensibilidad de los estudiantes hacia aspectos relacionados con la educación ambiental y sobre la necesidad de su desarrollo. En todos los casos el mayor porcentaje de respuestas se ubicaron en las escalas del cuatro al cinco, o sea, de acuerdo o totalmente de acuerdo. Sólo esto no es así en el caso del ítem "Para resolver los problemas de un país relacionados con la protección del medio ambiente, ¿Es preferible invertir en innovación tecnológica y ciencia antes que en educación ambiental?"(P18) en el cual manifestaron estar totalmente en desacuerdo o en desacuerdo, lo que refleja también una actitud positiva hacia la educación ambiental (ver figura 2).

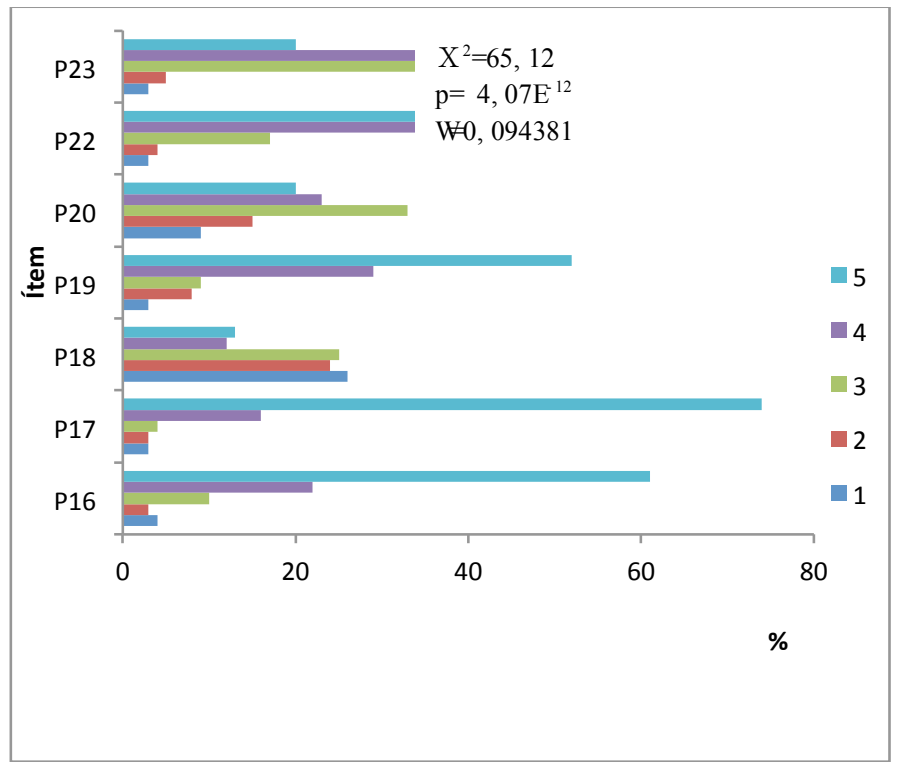

Figura 2: Percepción de los estudiantes sobre aspectos relacionados con la educación ambiental.

De forma general los rasgos generales concluyentes de las respuestas a estos ítems son:

- La educación ambiental debe ser una asignatura obligatoria en todos los niveles de enseñanza, incluso aunque esto presuponga dejar de recibir contenidos de cálculo, física, lenguas u otros más a tono con la preparación profesional. A su vez se destaca que para desarrollar la misma debe considerarse como un eje transversal en los contenidos, con sus actividades en los programas o currículos escolares.

- Todas las personas deben adquirir una sólida educación ambiental.

- Para resolver los problemas de un país relacionados con la protección del medio ambiente, la educación ambiental constituye una solución más eficaz incluso que la inversión en innovación 
tecnológica y ciencia.

- La génesis de una sólida cultura debe considerarse importante para el desarrollo de la educación ambiental.

- Según los valores calculados del coeficiente de concordancia de Kendal (W=0,094381) y sus parámetros: chi cuadrado $(\chi 2=65,12)$ y $p$ value $\left(p=4,07 E^{-12}\right)$ se rechaza la hipótesis nula que plantea que los encuestados no están de acuerdo en sus valoraciones lo cual indica que hay un alto nivel de concordancia en las respuestas dadas a estos ítem.

En el ítem 21 se les pidió a los encuestados relacionar las posibles acciones estratégicas que pudieran ser incluidas en programas de desarrollo de la educación ambiental en su universidad y se resalta el carácter posesivo de forma intencional (tabla4). Las acciones de mayor preferencia a realizarse por los estudiantes al aplicarse algún tipo de estrategia dirigida al fomento de la educación ambiental con frecuencias absolutas muy similares en las encuestas fueron el impulso a programas de reciclaje; el desarrollo de temas de educación ambiental mediante reuniones, eventos, festivales, publicidad diversa y el poder desarrollar proyectos de autogestión económica como reforestación, producción de abono orgánico, papel artesanal, reciclaje de papel, vidrio, aluminio y otros. En segundo lugar por el nivel de preferencia se ubicó la planificación y ejecución de actividades que permitan la participación estudiantil en la modificación del medio físico y natural. Con frecuencias absolutas más bajas y similares entre si se ubicaron la imposición de bonificaciones por buena conducta ambiental; la penalización por dañar el medio ambiente; el fomento de programas informativos y el desarrollo en asociación con los organismos regionales de proyectos de ecoturismo, ecofolklore y ecociencia.

Tabla 4. Acciones estratégicas propuestas a realizarse en programas de educación ambiental en la Universidad Politécnica del Valle del Évora, México.

\begin{tabular}{|c|c|}
\hline Acciones estratégicas & $\begin{array}{l}\text { Frecuencia } \\
\text { absoluta }\end{array}$ \\
\hline Impulsar programas de reciclaje. & 56 \\
\hline $\begin{array}{l}\text { Desarrollar temas de educación ambiental mediante } \\
\text { reuniones, eventos, festivales, publicidad diversa. }\end{array}$ & 55 \\
\hline $\begin{array}{l}\text { Desarrollar proyectos de autogestión económica como } \\
\text { reforestación, producción de abono orgánico, papel } \\
\text { artesanal, reciclaje de papel, vidrio, aluminio y otros. }\end{array}$ & 52 \\
\hline $\begin{array}{l}\text { Planificar y ejecutar actividades que permitan la } \\
\text { participación estudiantil en la modificación del medio } \\
\text { físico y natural. }\end{array}$ & 41 \\
\hline $\begin{array}{l}\text { Desarrollar contenidos de educación ambiental a la vez } \\
\text { que se adquieren conocimientos sobre la profesión } \\
\text { buscando los puntos comunes. }\end{array}$ & 24 \\
\hline Bonificaciones por buena conducta ambiental. & 17 \\
\hline Penalizar la falta de concientización ambiental. & 14 \\
\hline Fomentar programas informativos. & 13 \\
\hline $\begin{array}{l}\text { Desarrollar en asociación con los organismos regionales } \\
\text { proyectos de ecoturismo, ecofolklore y ecociencia. }\end{array}$ & 12 \\
\hline
\end{tabular}


Crear grupos de estudiantes interesados en la 1

educación ambiental y desarrollar estrategias que impulsen la conservación del medio ambiente, que sea considerada como una extra-clase para así esto genere más interés en los estudiantes.

Se agregó por parte de un encuestado una acción a la lista ofrecida que consistió en la creación de grupos estudiantiles interesados en la educación ambiental que desarrollen acciones de protección del medio ambiente y que estas actividades constituyen motivo de bonificación en su currículo aun cuando se desarrolle de forma extracurricular, lo cual puede generar mayor interés para su ejecución.

En casi todos los casos se observa como rasgo común que las acciones propuestas por los estudiantes tienen carácter dinámico, pues solo una que fue preferida por 23 de los encuestados se concibe "dentro del aula" que es la referente a "desarrollar contenidos de educación ambiental a la vez que se adquieren conocimientos sobre la profesión buscando los puntos comunes", aunque la enseñanza universitaria actual no se concibe sin una estrecha vinculación de contenidos teóricos con los de tipo práctico por lo que este tipo de actividad puede y debe desarrollarse en espacios exteriores relacionados con la especialidad. Dígase por ejemplo un área de producción agropecuaria para el caso de los estudiantes de Agrotecnología.

Lo anterior debe ser considerado por la dirección de la universidad, así como por los grupos de profesores, las acciones que se conciban para ser atractivas deben ser de tipo práctico, novedosas deben salirse del espacio físico del aula y pueden ser aprovechados los accidentes geográficos de cada zona, la actividad económica, cultural, recreativa que se desarrolla en las propias comunidades para ponerlas en función de motivar interés por la educación ambiental. Deben revisarse los programas analíticos de cada asignatura de tal forma que se encuentren los puntos comunes entre las actividades curriculares y el impacto al medio ambiente que generan las actividades que en ellas se explican para no convertir la educación ambiental en una asignatura más sino en parte de cada una de ellas.

La Conferencia Intergubernamental sobre Educación Ambiental en Tbilisi (1977), estableció que ésta debía adoptar una perspectiva holística que incluyera la dimensión sociocultural, ecológica, política, económica y demás, a fin de propiciar la solución de problemas específicos. Otros instrumentos internacionales con posterioridad también advirtieron la problemática como la Declaración de Río de 1992 y recientemente el acuerdo de París un instrumento de alcance mundial para enfrentar de manera global el cambio climático, el cual busca que por lo menos 195 países reorienten su desarrollo hacia un mundo más sostenible, con menores emisiones y con capacidad de adaptarse a un clima más extremo (Imco, 2017). Además de ello, sugiere que los contenidos, métodos y materiales específicos se adapten a las necesidades de los educandos. A tenor con las respuestas dadas a los ítems 25 y 26 en los que se les preguntó acerca de si los programas de educación ambiental deben regirse por programas propios elaborados según las características de cada territorio o estos deben derivarse de los orientados desde la Secretaría de Educación, los estudiantes mayoritariamente expresaron preferencia porque la misma se rija para su desarrollo por programas específicamente elaborados en las universidades que reflejen las características de cada territorio. Por último en el ítem 26 se les pidió valorar el grado de importancia de los diferentes sectores de la sociedad 
para encarar la gestión de la educación ambiental al preguntarles "En la siguiente escala valorativa indique del 1 al 5, (siendo 1 el más importante y 5 el menos importante), los sectores que considere tienen mayor responsabilidad en gestionar, promover y difundir la educación ambiental". Los sectores relacionados fueron:

- Sector educativo.

- Sector empresarial.

- Sector gubernamental.

- Familia.

- Medios de comunicación.

Como resultado se obtuvo (figura 3) que la familia fue citada por más del $70 \%$ como la de mayor responsabilidad en la tarea y en segundo lugar con porcentajes de respuestas cercanos al $50 \%$ se fueron referidos el sector gubernamental, los medios de comunicación y el sector educativo. El sector empresarial fue señalado en el $40 \%$ de las respuestas como el más importante o importante. Los otros sectores referidos fueron señalados como menos importante en el rango del 5 al $10 \%$ de las respuestas, destacan la relevancia de que la protección del medio ambiente y la gestión de la educación ambiental debe ser tarea de todos en la sociedad para lograr éxito.

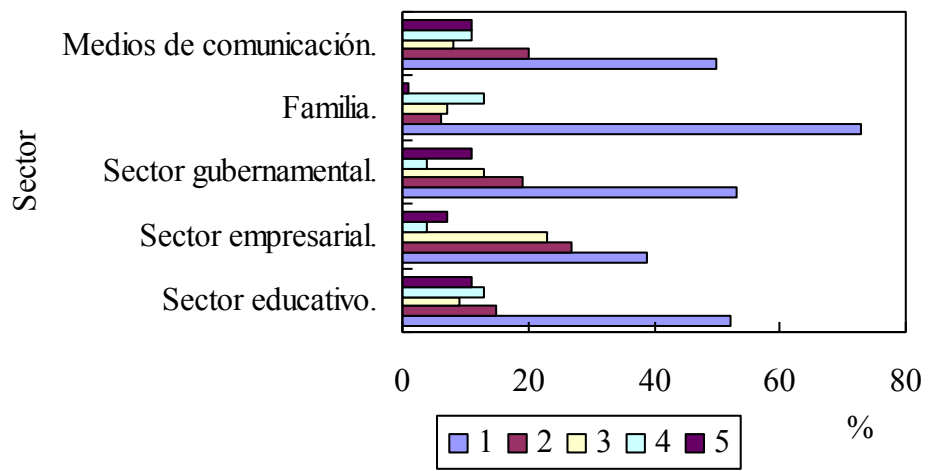

Figura 3. Percepción sobre grado de responsabilidad de diferentes sectores de la sociedad para gestionar la educación ambiental.

\section{CONCLUSIONES}

En este trabajo se llegó a las siguientes conclusiones:

1. El potencial de las Instituciones de Educación Superior, considerando sus funciones centrales, deberán incidir de manera importante en la promoción, difusión y concientización de la comunidad universitaria en temas de educación ambiental.

2. La percepción de los estudiantes muestra un interés relevante en la participación como entes activos para promover acciones y estrategias dirigidas al fomento de la educación ambiental, con una frecuencia absoluta fueron el impulso a programas de reciclaje; el desarrollo de temas de educación ambiental mediante reuniones, eventos, festividades, publicidad diversa, así como desarrollo de proyectos de autogestión económica como reforestación, producción de abono orgánico, papel artesanal, reciclaje de papel, vidrio, aluminio y otros. 


\section{BIBLIOGRAFIA}

Anuies (Asociación Nacional de Universidades e Instituciones de Educación Superior). (2009). Plan de acción para el desarrollo sustentable en las instituciones de educación superior. México. Recuperado PlandeAccionSemarnat.pdf de:http://www.complexus.org.mx/Documentos/ANUIES-

Ávila, A. (2009).La educación ambiental a nivel superior: Centro de Investigación y Estudios de Posgrado. México. Recuperado de: http://www.bvsde..paho..org/bvsaidis/ mexico13/052. Pdf

Barazarte, R. Castro, A. N., Vallejo, F. \&García, P. (2014). El conocimiento ambiental y el comportamiento proambiental de los estudiantes de la Enseñanza media, en la Región de Valparaíso (Chile).Revista de Educación, 12-34. DOI: 10.4438/1988-592X-RE-2014-364-255

Bedoy, V. (2009). La historia de la educación ambiental: reflexiones pedagógicas. México. Recuperado de: http://www.quadernsdigitals.net/datos/hemeroteca/r_24/nr_284/a_3672/3672.html

Bravo, M.T. (2009). Las instituciones de educación superior se organizan para participar en el cambio ambiental. México. Recuperado de: http://anea.org.mx/docs/BravoLasIESseorganizan.pdf

Calafell, G. \& Gargallo, J.B. (2014). Identificación y caracterización de las concepciones de medio ambiente de un grupo de profesionales de la educación ambiental. Revista Enseñanza de las Ciencias, 32(3), 205-225. DOI: https://doi.org/10.5565/rev/ensciencias.1048

Espino, P., Olaguez, E. \&Davizon, Y.A. (2015). Análisis de la Percepción del Medio Ambiente de los Estudiantes de Ingeniería en Mecatrónica. Revista Formación Universitaria, 8(4), 45-54. DOI: https://dx.doi.org/10.4067/S0718-50062015000400006

García, P. \&Navarro, M. (2011). Qué piensan y cómo dicen que actúan los alumnos y profesores de un Centro de Educación Secundaria sobre la gestión del agua, la energía y los residuos. Revista Enseñanza de las Ciencias, 29(1), 061-074.

Imco (Instituto Mexicano para la Competitividad). (2017). México. Recuperado en: http://imco.org.mx/medio_ambiente/mexico-ratifica-el-acuerdo-de-paris-sobre-el-cambioclimatico/

González, E. (2011). Otra lectura a la historia de la educación ambiental en América Latina y el Caribe. Tópicos en educación ambiental. Desenvolvimento e Meio Ambiente. 3, 141-158.

LGEEPA (Ley General del Equilibrio Ecológico y La Protección al Ambiente) (2008). México. Recuperado en: http://www.diputados.gob.mx/LeyesBiblio/pdf/148.pdf (9/09/2009).

Márquez, R.I., Salavarría, O.O., Eastmond, A. M., Ayala, E., Arteaga, M.A., Isaac-Márqueza. P., Sandoval, J.L. \&Manzanero, L.A. (2011). Cultura ambiental en estudiantes de bachillerato: Estudio de caso de la educación ambiental en el nivel medio superior de Campeche. Revista Electronica de Investigación Educativa, 13(2) ,83-99.

Medina, L. (2009). La Educación Ambiental en el Nivel Superior. México. Recuperado de: http://www.uaemex.mx/plin/psus/rev3/medina.pdf

Novo, M. \&Bautista, M.J. (2012). Análisis de la incidencia de la educación ambiental para el 
desarrollo sostenible en las revistas científicas españolas. Revista de Educación, 358, 583597.

Olaguez, E. \&Espino, P. (2013). Perspectivas de educación ambiental en las instituciones de educación superior. Revista Iberoamericana de las Ciencias Biológicas y Agropecuarias, 2(3).

Pnuma (Programa de las Naciones Unidas para el Medio Ambiente) (2009). Oficina Regional para América Latina y el Caribe. Recuperado en: http://www.pnuma.org/docamb/mh1972.php.

Sosa, M., Alcalá, J., Soto, R., Lebgue, T., \& Quintana, C. (2008). Percepción ambiental de estudiantes universitarios a través de variables medioambientales. Revista Latinoamericana de Recursos Naturales, 4(2), $178-184$.

Sosa, S.B., Márquez, R.I., Eastmond, R.A., Ayala, M.E., \&Arteaga, M.A. (2010). Educación superior y cultura ambiental en el sureste de México. Universidad y ciencia, 26(1), 33-49.

Unesco (Organización de las naciones unidas para la educación, la ciencia y la cultural) (2010). Recuperado en http://portal.unesco.org/es/ev.phpRL_ID=29011\&URL_DO=DO_TOPIC\&URL_SECTION=201.html

Zamorano, B., Parra V., Peña, F., Castillo, Y., \&Vargas, J.I. (2009). Percepción ambiental en estudiantes de secundaria. Revista Electrónica Actualidades Investigativas en Educación, 9(3) ,1-19. 